\title{
Numerical Simulations of Bromsulphthalein Test for Human Liver
}

\author{
A. A. M. Arafa*, I. M. Hanafy and M. I. Gouda \\ Department of Mathematics and Computer Science, Faculty of Science, Port Said University, Egypt
}

Received: 6 Nov. 2013, Revised: 11 Feb. 2014, Accepted: 12 Feb. 2014

Published online: 1 May 2014

\begin{abstract}
In this paper, we introduce the model of Bromsulphthalein (BSP) test whose components are BSP in blood, BSP in liver and BSP in bile with fractional order. Generalized Euler method (GEM) is performed to solve the problem. We compare fractional results and integer results with clinical data. The results show that the fractional order is closer than clinical data than standard model.
\end{abstract}

Keywords: Generalized Euler method (GEM), BSP model, Fractional Calculus, Numerical solution.

\section{Introduction}

A mathematical model is a description of a system using mathematical concepts and language. A model may help to explain a system and to study the effects of different components, and to make predictions about behavior. These models play an important role in investigation of metabolic endocrine system kinetics in human body. The mathematical model should be done in such a way that data given by clinical experiments on humans would give relevant information on the status of individual. The Bromsulphthalein (BSP) test is used to assess liver function. BSP is a hepatotropy matter, which is injected into the blood. The liver is only organ in the body which takes BSP and secretes it directly into the bile [1]. Different methods were introduced to get approximate analytical solution or numerical solution for BSP test [ 2 ]. First we analyze simplified model which describes the extraction of BSP in blood, liver and bile that introduced in [2]. There are three components: $x$, the amount of BSP in blood $y$, the amount of BSP in liver and $z$, the amount of BSP in bile at time $t$. Then the following ordinary differential equation (ODE) describe the evolution of the system:

$$
\begin{gathered}
\frac{d x}{d t}=-a x+b y, \\
\frac{d y}{d t}=a x-(b+d) y, \\
\frac{d z}{d t}=d y .
\end{gathered}
$$

Where

$a=0.0547241, b=0.0152577$ and $c=0.00939036$ are transfer rates which determined in [1].

Now, we introduce a general model for BSP test as follows

$$
\begin{gathered}
D^{\alpha}(x)=-a x+b y, \\
D^{\alpha}(y)=a x-(b+d) y, \\
D^{\alpha}(z)=d y .
\end{gathered}
$$

Where $0<\alpha<1$

With initial conditions

$$
x(0)=250, y(0)=0, z(0)=0 .
$$

The rest of paper is organized as follows. In section 2, we introduce the fractional calculus theory. In section 3 we show the idea of Generalized Taylor formula .In section 4, we introduce generalized Euler method (GEM) for solving fractional order ordinary differential equations. In section5, conclusion is presented. Numerical results of GEM, clinical data and compare between them are presented in section5. in section 6 conclusion is presented.

\section{Fractional calculus}

The field of fractional calculus is almost as old as calculus itself, but over the last decades the usefulness of

\footnotetext{
*Corresponding author e-mail: anaszi2@yahoo.com
} 
this mathematical theory in applications as well as its merits in pure mathematics has become more and more evident $[3,4]$. Fractal differential equations have attracted many researchers due to their important applications in fluid flow, mechanics, biology, physics, epidemiology and engineering, and other applications. This is because of the fact that the realistic modeling of a physical phenomenon does not depend only on the instant time, but also on the history of the previous time which can also be successfully achieved by using fractional calculus. In other words, previous values of the solution and the derivatives in fractional order differential equations are required to obtain a solution at a particular instance. The memory effect of the convolution in the fractional integral gives the equation increased expressive power. There are several definitions of a fractional derivative of $\alpha>0$ [5]. The two most commonly used definitions are Riemann-Liouville and Caputo. Each definition uses Riemann-Liouville fractional integration and derivatives of whole order. The difference between the two definitions is in order of evaluation.

Definition2.1Riemann Liouville fractional integration of order $\alpha$ is defined as:

$$
\begin{gathered}
J^{\alpha} f(x)=\frac{1}{\Gamma(\alpha)} \int_{0}^{x}(x-t)^{\alpha-1} f(t) d t, \quad \alpha>0, x>0 \\
J^{0} f(x)=f(x)
\end{gathered}
$$

Definition 2.2 The Caputo fractional derivative of the power function satisfies [6] :

$D_{*}^{\alpha} t^{p}=\left\{\begin{array}{cc}\frac{\Gamma(p+1)}{\Gamma(p-\alpha+1)} t^{p-\alpha}=D^{\alpha} t^{p} & , n-1<\alpha<n, p>n-1, p \in R \\ 0 & , n-1<\alpha<n, p \leq n-1, p \in R\end{array}\right.$

Definition 2.3.Riemann-Liouville and Caputo fractional derivatives of order $\alpha$ can be defined respectively as :

$$
\begin{aligned}
& D^{\alpha} f(x)=D^{m}\left(J^{m-\alpha} f(x)\right), \\
& D_{*}^{\alpha} f(x)=J^{m-\alpha}\left(D^{m} f(x)\right),
\end{aligned}
$$

whrere

$$
m-1<\alpha \leq m, m \in N
$$

Properties of the operator $D_{*}^{\alpha}$ can be found in [13]. We mention only the following:

(1) Interpolation

$$
\lim _{\alpha \rightarrow n} D_{*}^{\alpha} y(t)=y^{(n)}(t),
$$

(2) Linearity

$$
D_{*}^{\alpha}(a y(x)+b z(t))=a D_{*}^{\alpha} y(x)+b D_{*}^{\alpha} z(t) .
$$

(3) Commutation

$$
D_{*}^{\alpha} D^{m} f(t)=D_{*}^{\alpha+m} f(t) .
$$

The definition of fractional derivative involves an integration which is non-local operator (as it is defined on an interval) so fractional derivative is a non-local operator. In other word, calculating time-fractional derivative of a function $f(t)$ at some time $t=t_{1}$ requires all the previous history, i.e. all $f(t)$ from $t=0$ to $t=t_{1}$. Many mathematicians have tried to study some models of infectious diseases models using the fractional calculus. The reason behind using fractional ordinary differential equation (FODE) is that (FODE) is related to in most biological systems with memory. It gives us more approximate solution to this systems and more general nature $[12,13,14,15,16,17,18,19,20,21]$.

\section{Generalized Taylor's formula}

In this section we introduce a generalization of Taylor's formula that involves Caputo fractional derivatives. This generalization is presented in [7].

Suppose that $D_{*}^{k \alpha} f(x) \in C(0, a]$, for $k=0,1, \ldots, n+$ 1 , where $0<\alpha \leq 1$. Then we have

$f(x)=\sum_{i=0}^{n} \frac{x^{i \alpha}}{\Gamma(i \alpha+1)}\left(D_{*}^{i \alpha}\right)\left(0_{+}\right)+\frac{\left(D_{*}^{(n+1) \alpha} f\right)(\zeta)}{\Gamma((n+1) \alpha+1)} x^{(n+1) \alpha}$,

With $0<\zeta<x \in(0, a]$

In case of $a=1$, the generalized Taylor's formula (2) reduces to the classical Taylor's formula.

\section{Generalized Euler method (GEM)}

Most nonlinear fractional differential equations do not have analytic solutions, so approximations and numerical techniques must be used [8,9]. The decomposition method (ADM), the variational iteration method (VIM), and The homotopy analysis method (HAM) are relatively new approaches to provide an analytical approximate solution to linear and nonlinear problems, and they are particularly valuable as tools for scientists and applied mathematicians, because they provide immediate and visible symbolic terms of analytic solutions, as well as numerical approximate solutions to both linear and non-linear differential equations $[3,4]$. In recent years, the application of the ADM, VIM, in linear and nonlinear problems has been developed. On the other hand, these methods are effective for small time, i.e $t \ll 1$, however such methods cannot solve the problem for larger time and in fact the solution of the chaotic system using HPM is an open problem. Nevertheless by chance, there are cases at which these methods give good approximation for a large range of time ( $\mathrm{t}$ ). A few numerical methods for fractional differential equations models of infectious diseases models have been presented in the literature. However many of these methods are used for very specific types of differential equations, often just linear 
equations or even smaller classes. In[10], Odibat and Momani derived the generalized Euler's method that we have developed for the numerical solution of initial value problems with Caputo derivatives. The method is a generalization of the classical Euler's method. In this paper, GEM is used to obtain numerical solution of fractional order model of BSP test .They apply (GEM) to study the (BSP) test model. Consider the initial value problem

$$
\left(D_{*}^{\alpha} y(t)=f(t, y(t)), y(0)=y_{0}, 0<\alpha \leq 1, t>0 .\right.
$$

Let $[0, a]$ be the interval over which we want to find the solution of problem (3).in actuality, we will not find function $y(t)$ that satisfies value problem Eq. (3).instead ,asset of points $\left\{t_{j}, y\left(t_{j}\right)\right\}$ is generated, and the points are used for our approximation .for convenience we subdivide the interval $[0, a]$ into $k$ subintervals $\left[t_{j}, t_{j+1}\right]$ of equal width $h=\frac{a}{k}$ by using the nodes $t_{j}=j h$,for $j=$ $0,1, \ldots \ldots, k$. Assume that $y(t), D_{*}^{\alpha} y(t)$ and $D_{*}^{2 \alpha} y(t)$ are continuous on $[0, a]$ and use the generalized Taylor's formula Eq. (2) to expand $y(t)$ about $t=t_{0}=0$. For each value $t$ is a value $c_{1}$ so that $[11,12]$

$y(t)=y\left(t_{0}\right)+\left(D_{*}^{\alpha} y(t)\right)\left(t_{0}\right) \frac{t^{\alpha}}{\Gamma(\alpha+1)}+\left(\left(D_{*}^{2 \alpha} y(t)\right)\left(c_{1}\right) \frac{t^{2 \alpha}}{\Gamma(2 \alpha+1)}\right.$.

When $\left(D_{*}^{\alpha} y(t)\right)\left(t_{0}\right)=f\left(t_{0}, y\left(t_{0}\right)\right)$ and $h=t_{1}$ are substituted Eq.(4),the result is an expression for $y\left(t_{1}\right)$ :

$y(t)=y\left(t_{0}\right)+f\left(t_{0}, y\left(t_{0}\right)\right) \frac{h^{\alpha}}{\Gamma(\alpha+1)}+\left(\left(D_{*}^{2 \alpha} y(t)\right)\left(c_{1}\right) \frac{h^{2 \alpha}}{\Gamma(2 \alpha+1)}\right.$.

If the step size $h$ is chosen small enough, then we may neglect the second -order term (involving $h^{2 \alpha}$ ) and get

$$
y\left(t_{1}\right)=y\left(t_{0}\right)+\frac{h^{\alpha}}{\Gamma(\alpha+1)} f\left(t_{0}, y\left(t_{0}\right)\right),
$$

And so on ,we get $y\left(t_{1}\right), \quad y\left(t_{2}\right), \ldots \ldots$, the process is repeated and generates a sequence of points that approximates the solution,then we can get the general formula for generalized Euler's method (GEM) when $t_{j+1}=t_{j}+h$ as follow [10]

$$
y\left(t_{j+1}\right)=y\left(t_{j}\right)+\frac{h^{\alpha}}{\Gamma(\alpha+1)} f\left(t_{j}, y\left(t_{j}\right)\right)
$$

For $j=0,1, \ldots \ldots, k-1$. it is clear if $\alpha=1$, then the generalized Euler's method (5) reduces to the classical Euler's method this method discuss in details in [10].

\section{Numerical result}

Now we solve the BSP model by using (GEM) method and compare between our data and clinical data in [1] .we show the accuracy of GEM method in following figures. The results in table 1 show that the amount of BSP in blood is decreased by the time. The results in fractional order when $\alpha=0.91$ is closer than clinical data than the results when $\alpha=1$. The results in table 2 show that the amount of BSP in bile is increased by the time. The results when $\alpha=0.91$ is closer than clinical data than results when $\alpha=$ 1 .

Table 1: The numerical results of $x(t)$.

\begin{tabular}{|l|l|l|l|}
\hline$t$ & $\begin{array}{l}\alpha=1 \\
\text { GEM }\end{array}$ & $\begin{array}{l}\alpha=0.91 \\
\text { GEM }\end{array}$ & $\begin{array}{l}\text { Clinical } \\
\text { data [1] }\end{array}$ \\
\hline 0 & 250 & 250 & 250 \\
\hline 3 & 223.5900 & 222.6800 & 221 \\
\hline 5 & 190.4940 & 188.6686 & 184 \\
\hline 10 & 148.9473 & 146.4065 & 141 \\
\hline 20 & 99.1009 & 96.5784 & 98 \\
\hline 30 & 73.5816 & 71.6031 & 80 \\
\hline 43 & 56.7835 & 55.4000 & 64 \\
\hline
\end{tabular}

Table 2: The numerical results of $z(t)$.

\begin{tabular}{|l|l|l|l|}
\hline $\mathrm{t}$ & $\begin{array}{l}\alpha=1 \\
\text { GEM }\end{array}$ & $\begin{array}{l}\alpha=0.91 \\
\text { GEM }\end{array}$ & $\begin{array}{l}\text { Clinical } \\
\text { data [1] }\end{array}$ \\
\hline 0 & 0 & 0 & 0 \\
\hline 20 & 14.4380 & 15.4559 & 10.5 \\
\hline 40 & 41.5468 & 43.8969 & 34 \\
\hline 60 & 68.3305 & 71.5811 & 63.8 \\
\hline 80 & 98.5566 & 97.7726 & 92.7000 \\
\hline 100 & 113.2136 & 117.3576 & 117.0000 \\
\hline 120 & 131.3963 & 135.7053 & 136.3000 \\
\hline 140 & 147.1688 & 151.5200 & 152.1000 \\
\hline 150 & 154.2508 & 158.5872 & 159.2000 \\
\hline
\end{tabular}

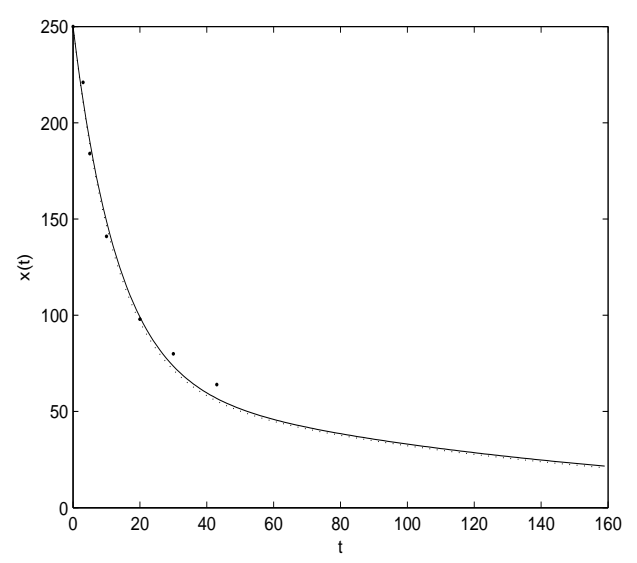

Fig. 1: The amount of BSP in blood $x(t)$ : solid line represent data from GEM method when $\alpha=1$, dotted line represent data from GEM method when $\alpha=0.91$ and dots are clinical data 


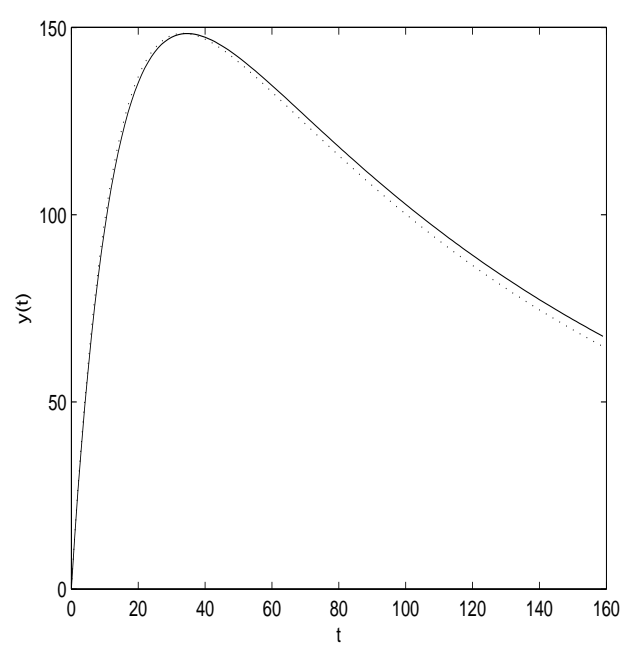

Fig. 2: The amount of BSP in blood $y(t)$, solid line represent data from GEM method when $\alpha=1$, dotted line represent data from GEM method when $\alpha=0.91$ and dots are clinical data.

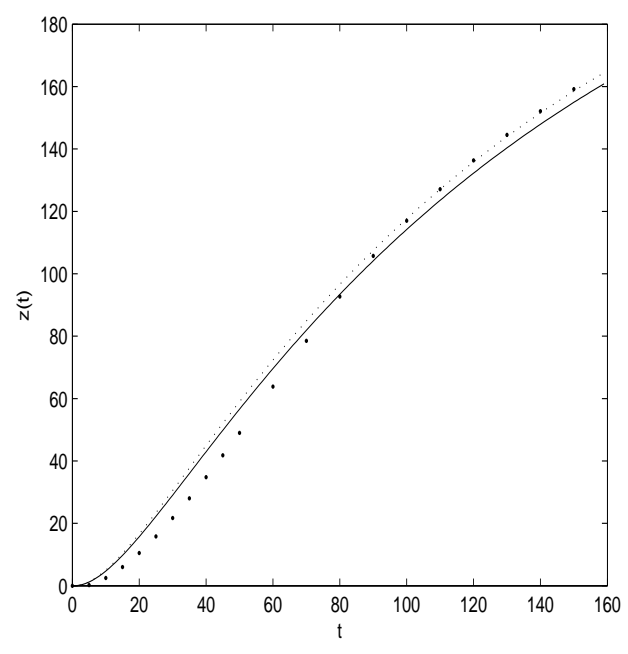

Fig. 3: The amount of BSP in bile $z(t)$ : solid line represent data from (GEM) method at $\alpha=1$, dotted line represent data from (GEM) method at $\alpha=0.91$ and dots are clinical data.

\section{Conclusion}

In this paper, we use generalized Euler method (GEM) in fractional order to solve BSP test model . In Figures 1,2,3 and Tables 1,2 show that the comparisons between the results of $(\mathrm{GEM})$ when alpha symbol $=1$, Alpha symbol in fractional order and clinical data. The results show that (GEM) method in fractional order is closer than classical (GEM) method to clinical data. The above method can solve nonlinear cases with no difficulty and more accuracy

\section{References}

[1] L . elechovsk : A simple mathematical model of the human liver, Appl.Math., 49, 227-246 (2004).

[2] J. M. Watt, A. Young : An attempt to simulate the liver on a computer. J., 5, 221-227 (1962).

[3] A.M.A. El-Sayed, S.Z. Rida, A.A.M. Arafa, On the solutions of time-fractional bacterial chemotaxis in a diffusion gradient chamber, Int. J. Nonlinear Sci., 7, 485492 (2009)

[4] I. Hashim, O. Abdulaziz, S. Momani, Homotopy analysis method for fractional IVPs, Commun. Nonlinear Sci. Numer. Simul., 14, 674-684 (2009).

[5] Z. Odibat, S. Momani, H. Xu, A reliable algorithm of homotopy analysis method for solving nonlinear fractional differential equations, Appl. Math Model, 34, 593-600 (2010).

[6] R. Goren o and F. Mainardi, Essentials of fractional calculus, Preprint submitted to MaPhySto Center, January 28, (2000).

[7] Z. Odibat, N. Shawagfeh, Generalized Taylor's formula, Appl. Math. Comput., 186, 286-293 (2007)

[8] M. Zurigat, S. Momani, A. Alawneh, Analytical approximate solutions of systems of fractional algebraic differential equations by homotopy analysis method, Comput. Math. Appl., 59, 1227-1235 (2010).

[9] M. Zurigat, S. Momani, Z. Odibat, A. Alawneh, The homotopy analysis method for handling . systems of fractional differential equations, Appl. Math Model, 34, 2435 (2010).

[10] Z. Odibat, S. Moamni, An algorithm for the numerical solution of differential equations of fractional order, J. Appl. Math. Informat., 26, 15-27 (2008).

[11] A.A.M. Arafa , S.Z. Rida , M. Khalil, The effect of antiviral drug treatment of human immunodeficiency virus type 1 (HIV-1) described by a fractional order model, Applied Mathematical Modelling, 37, 2189-2196 (2013).

[12] K. Diethelm , N.J. Ford , A.D. Freed , Yu. Luchko, Algorithms for the fractional calculus: Aselection of numerical methods , Comput. Methods Appl. Mech. Engrg., 194, 743-773 (2005).

[13] A. A. M. Arafa, Fractional Differential Equations in Description of Bacterial Growth, Differential Equations \& Dynamical Syststem, 21, 205-214 (2013).

[14] A.A. M. Arafa, S.Z. Rida, M. Khalil, Approximate analytical solutions of Schnakenberg systems by homotopy analysis method, Applied Mathematical Modeling, 37, 2189-2196 (2013).

[15] A.A.M. Arafa,S.Z. Rida, A.A. Mohammadein, and H.M. Ali, Solving Nonlinear Fractional Differential Equation by Generalized Mittag-Leffler Function Method, Commun. Theor. Phys., 59, 661-663 (2013). 
[16] A.A. M. Arafa, S.Z. Rida, Numerical modeling for some generalized coupled nonlinear evolution equations, Mathematical and Computer Modelling, 56, 268-277 (2012).

[17] A.A. M. Arafa, S.Z. Rida, H. Mohamed, Approximate analytical solutions of Schnakenberg systems by homotopy analysis method, Applied Mathematical Modelling, 36, 4789-4796 (2012).

[18] A. A. M. Arafa, 2011. Series Solutions of Time-Fractional Host-Parasitoid Systems, J Stat Phys, 145, 1357-1367 (2011).

[19] S. Z. Rida and A. A. M. Arafa, New Method for Solving Linear Fractional Differential Equations, International journal of differential equations, 2011, Article ID 814132, 8 pages.

[20] A. A. M. Arafa, S. Z. Rida, H. Mohamed, Homotopy Analysis Method for Solving Biological Population Model, Commun. Theor. Phys., 56, 797-800 (2011).

[21] A.A.M. Arafa , S. Z. Rida , M.Khalil, Fractional modeling dynamics of $\mathrm{HIV}$ and $\mathrm{CD} 4^{+}$T-cells during primary infection, Nonlinear Biomedical Physics, 6, 1 (2012)

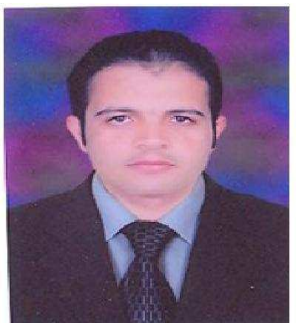

A. A. M. Arafa is a lecturer of Mathematics at Port Said University, Faculty of Science. He is referee and Editor of several international journals in the frame of pure and applied mathematics. His main research interests are: Fractional Calculus and applications.

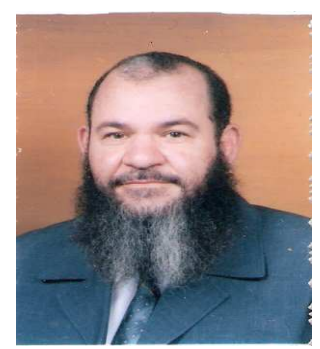

I. M. I. Hanafy $\mathrm{Ph}$. D. in Mathematics ( Fuzzy Topology ) from the Faculty of Science, Tanta University , Egypt, 1990. he is Professor and Head of Department of Mathematics, Faculty of Science, Portsaid University, Portsaid, Egypt.his research interesrs in the areas of Fuzzy Topological Spaces, Intuitionistic Fuzzy Topological Spaces,Fuzzy Relations and Genuine Topological Spaces.

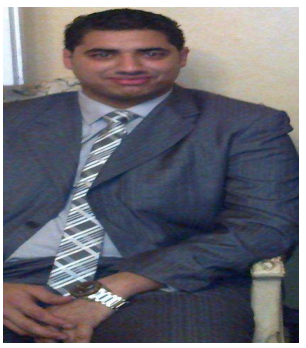

Mohammed Ibrahem Gouda B. Sc. in Mathematics from the Faculty of Science, Port-Said University, Egypt, 2012. His research interests are in the areas of fractional calculas in biological modeling . 